\title{
Diccionario ilustrado de algunos términos utilizados en zoología, una vía novedosa para su aprendizaje
}

\author{
Dictionary of some terms used in zoology, a new way for its learning
}

Recibido: julio 30 de 2020 | Revisado: agosto 12 de 2020 | Aceptado: setiembre 02 de 20202

\author{
Rafael Arminaña-García ${ }^{\mathrm{I}}$ \\ Rigoberto Fimia-Duarte ${ }^{2}$ \\ José IANNACONE ${ }^{3,4}$ \\ Lorena Alvariño \& \\ YANIRA ZAITA-FERRER ${ }^{2}$
}

\section{RESUMEN}

El trabajo investigativo consistió en la elaboración de un diccionario ilustrado de algunos términos más utilizados en Zoología en griego o latín y su etimología en espańol. La obra está dirigida fundamentalmente a los estudiantes que cursan estudios en la carrera de Licenciatura en Educación-Biología en la Universidad Central "Marta Abreu», de Las Villas, Cuba. La selección de los términos que se han incluido en este diccionario va más allá de lo que se pudiera entender como zoológicos propiamente dicho, partiendo de la opinión de que todos coadyuvan al entendimiento cabal de la Zoología. Se hizo corresponder las ilustraciones con los requerimientos pedagógicos e higiénicos, contribuyendo de esta manera a la educación estética de los estudiantes y a elevar el interés por el material didáctico y se tomó en consideración las sugerencias y argumentaciones realizadas por diferentes expertos que fueron en gran medida la base para la realización de la obra desde el punto de vista de su concepción metodológica. La información para el diagnóstico es obtenida a partir de la aplicación de diferentes métodos empíricos como encuestas, entrevistas, observación y revisión de documentos. La obra se somete para su valoración a criterio de expertos y se implementó en los cursos académicos 2018 - 2020, con resultados satisfactorios.

Palavras chave: diccionario, etimología, conceptos, proceso de enseñanza-aprendizaje, zoología

\begin{abstract}
The research work consisted in the elaboration of an illustrated dictionary of some of the most used terms in Zoology in Greek or Latin and their etymology in Spanish. The work is aimed primarily at students studying in the Bachelor of Education career-Biology at the Central University "Marta Abreu", Las Villas. The selection of the terms that have been included in this dictionary goes beyond what could be understood as zoos properly speaking, based on the opinion that they all contribute to the complete understanding of Zoology. The illustrations were matched with the pedagogical and hygienic requirements, thus contributing to the aesthetic education of the students and raising interest in the didactic material and the suggestions and arguments made by different experts were taken into consideration, which were largely the basis for the realization of the work from the point of view of its methodological conception. The information for the diagnosis is obtained from the application of different empirical methods such as surveys, interviews, observation and document review. The work is submitted for evaluation at the discretion of experts and was implemented in the academic years 2018 - 2020, with satisfactory results.
\end{abstract}

Key words: concepts, dictionary, etymology, teaching-learning process, zoology

(C) Los autores. Este artículo es publicado por la Revista Campus de la Facultad de Ingeniería y Arquitectura de la Universidad de San Martín de Porres. Este artículo se distribuye en los términos de la Licencia Creative Commons Atribución No-comercial - Compartir-Igual 4.0 Internacional (https://creativecommons.org/licenses/ CC-BY), que permite el uso no comercial, distribución y reproducción en cualquier medio siempre que la obra original sea debidamente citada. Para uso comercial contactar a: revistacampus@usmp.pe. 


\section{Introducción}

En el perfeccionamiento del proceso de enseñanza-aprendizaje en la educación superior es inadmisible, si no se tiene en cuenta los medios de enseñanza que en él intervienen y sin considerar el importante rol que están llamados a desempeñar los profesores en relación con su uso y elaboración (Salinas, 2007; Kinchin, 2010; Latin et al., 2016, Armiñana et al., 2020).

La pedagogía contemporánea presta mucha atención a la metodología de enseñar a pensar y a trabajar con un creciente nivel de independencia. Es decir, que la docencia no puede limitarse a la simple transmisión de conocimientos por muy bien que esto se haga, sino a lograr también que los alumnos se apropien de métodos de trabajo con los que puedan abordar el estudio de las diferentes fuentes de conocimientos (Concepción, 2014; Ameyaw \& Kyere, 2019, Armiñana et al., 2020).

Se coincide con Armiñana (2014), que el valor pedagógico de los medios, brota más del contexto metodológico donde se usan, que de sus propias cualidades y posibilidades intrínsecas. Ese contexto es el que da su valor real, el que es capaz o no de dar juego a sus posibilidades técnicas para objetivos concretos, de forma que un mismo medio técnico puede tener una función muy distinta en una situación didáctica y en otra.

Para los autores de este trabajo, el impacto de las Tecnologías de la Información y la Comunicación (TIC) en la producción y difusión de medios de enseñanza y otros recursos didácticos es incuestionable. La docencia de calidad en la enseńanza superior ha de tenerlas en cuenta, ya que estas pueden presuponer un cambio esencial en la didáctica de cualquier asignatura.

En este sentido, la producción de recursos informativos y bibliográficos, la variedad y riqueza de sus contenidos, su incidencia en el aula y su función como transmisores de contenidos socialmente aceptados, hace que resulte interesante estudiar la contribución que realizan en la educación superior (Zumbado, 2004).

En América Latina, ocurre como regla que son las editoriales a través de su oferta de libros, las que programan los contenidos y en buena medida las actividades de aprendizaje en los centros de educación (Ferreira et al., 2000; Armiñana, 2014; Miller \& Harley, 2015; Holley, 2017). En Cuba por el contrario no se presenta este fenómeno y de forma general son los textos los que se ajustan, tal y como debe ser, a las exigencias y organización de las asignaturas.

Uno de los aspectos importantes en el proceso de enseńanza-aprendizaje de la Zoología y en cualquier ciencia, lo es sin lugar a duda la formación de conceptos. En la formación de ellos, se debe tener muy presente los antecedentes de otras asignaturas y niveles, siendo este factor más evidente cuando se trate de procesos cíclicos de conceptualización (Armiñana, 2019).

Para Moulines (2017), los conceptos son las unidades más básicas de toda forma de conocimiento humano, construcciones o autoproyecciones mentales, por medio de las cuales se comprenden las experiencias, es una unidad cognitiva de significado; un contenido mental que a veces se define como una unidad del conocimiento. 
A juicio de los autores de esta investigación, en el proceso de formación de conceptos, influyen también otros factores de gran valor, incluso algunos de ellos incontrolables en el orden didáctico como es, la experiencia científica técnica y pedagógica del profesor, o el nivel de autopreparacióny didactismodel estudiante y sus vivencias de índole personal. Hay que destacar que en la enseñanza superior la conceptualización alcanza su más alto nivel, pues se supone que allí el concepto del que se apropia el estudiante, no debe tener diferencias en profundidad con el que manejan los investigadores.

Un gran número de nombres técnicos pueden traducirse a sus equivalentes en idioma español, separando las palabras en partes. Muchos términos que se emplean en la ciencia despiertan también el interés general del lector medio y forzosamente, deben entenderse como parte de la cultura del pueblo y prueba de ello lo es el "Pequeño diccionario de términos biológicos" que incluye conceptos de genética, botánica, embriología, zoología, entre otros. Sin embargo, en la actualidad no se cuenta por lo menos que se conozca de un diccionario de términos utilizados con frecuencia en zoología.

Los autores de esta investigación han podido constatar desde una posición crítica, que para interpretar de forma precisa la etimología de la terminología técnica propia de la Zoología o usada comúnmente en ella, existen carencias evidenciadas en:

- Insuficiente conocimiento por parte de los estudiantes del latín o el griego, para poder interpretar el significado en idioma español de diferentes términos utilizados con mayor frecuencia en Zoología.

- Carencia de un diccionario ilustrado en formato electrónico de términos, cuyo origen de la palabra es el griego o el latín y que son comúnmente utilizados en Zoología.

- Escasa cognición de los prefijos y sufijos de origen griego o latín, para la construcción de un vocablo en idioma español de importancia en la elaboración de un concepto utilizado en Zoología y en particular en la nomenclatura científico - técnica.

Sean cuales fueran los planes de estudio en el proceso de enseñanza - aprendizaje de las asignaturas relacionadas con el estudio de los animales, en los objetivos generales, específicos, sistemas de contenidos y orientaciones metodológicas se hacía y se hace énfasis en la actualidad, en que los estudiantes deben dominar la etimología de diferentes términos utilizados en Zoología, así como la nomenclatura científica, según "International Code of Zoological Nomenclature" (ICZN) (Acosta, 2007; ICZN, 2020). Por ejemplo, en el programa de la Disciplina Zoología General se enfatiza en uno de los objetivos generales, se alude a que el estudiante debe desarrollar habilidades en la interpretación de la nomenclatura científica y en particular de los términos que provienen del idioma griego o latín, expresando de forma oral o escrita su significado en español (Banasco et al., 2016).

Asu vezen los programas delas asignaturas Zoología General I y II, respectivamente, se declaran como objetivos generales, que los estudiantes deben desarrollar habilidades en la interpretación de la nomenclatura científica y en particular de los términos que provienen del idioma griego o latín en la nomenclatura zoológica (Armiñana, 2016). 
Partiendo entonces de los presupuestos teóricos anteriormente planteados, surge el siguiente problema científico: ¿Cómo desarrollar habilidades intelectuales y prácticas en los estudiantes, para comprender el significado de la terminología zoológica que proviene del idioma griego o latín y su etimología en español? Para dar solución al problema y tomando en consideración lo planteado en párrafos anteriores relacionado con el uso de las TIC y la elaboración de nuevas bibliografías en formato digital, se plantea como objetivo de la investigación: proponer "Diccionario ilustrado de algunos términos utilizados en zoología".

\section{Materiales y métodos}

La investigación se realizó en la Facultad de Educación Media, con los estudiantes del $3^{\text {er }}$ año de la Licenciatura en Educación-Biología, de la Universidad Central "Marta Abreu" de Las Villas, Cuba. Para el desarrollo exitoso de esta investigación, se emplean métodos del nivel teórico y del nivel empírico, así como estadísticos y matemáticos, que permiten una comprensión integral del fenómeno estudiado.

Los métodos del nivel teórico:

- Histórico-lógico. Para analizar el proceder del problema de la investigación en los disímiles enfoques estudiados y el progreso de las soluciones propuestas.

- Analítico-sintético. Para valorar las principales contribuciones de estudiosos cubanos y extranjeros al tema de la investigación. Además, se conciertan y contrastan los criterios procedentes de las fuentes consultadas y en el análisis de los resultados del diagnóstico con el objetivo de orientar la estructura y organización del "Diccionario ilustrado de algunos términos utilizados en Zoología”.

- Inductivo-deductivo. Este método permitió, a partir de los instrumentos aplicados y las consultas bibliográficas realizadas, hacer inferencias alrededor de la situación real que poseen los estudiantes relacionados con el insuficiente conocimiento del latín o el griego, para poder interpretar el significado en idioma español de diferentes términos utilizados con mayor frecuencia en Zoología, sus necesidades y las formas de resolverlas, arribando a conclusiones sobre la forma de dar respuestas, mediante el diccionario.

- Sistémico-estructural. Para determinar los contenidos, exigencias, estructura y organización del diccionario y sobre la base de las relaciones de coordinación y subordinación de los conceptos que fundamentan los métodos $y$ procedimientos para el estudio de la Zoología.

- Ascenso de lo abstracto a lo concreto. Para el diseño y elaboración del diccionario, al revelar los elementos teóricos y prácticos necesarios.

- Modelación. Para representar teóricamente la concepción que sustenta la organización estructural del diccionario y en la construcción integral de este.

Métod.os del nivel empírico:

Análisisdedocumentos. Paraproporcionar la información necesaria del estado actual del objeto de investigación; como, por ejemplo, el análisis del Modelo del Profesional, programa de la disciplina y asignatura, respectivamente y otros.

- La observación. Para determinar las insuficiencias que poseen los estudiantes relacionados con la terminología 
científica técnica de la disciplina Zoología y apreciar cómo es la relación del estudiante con el diccionario.

- La encuesta. Para realizar una exploración a los estudiantes y profesores, sobre sus conocimientos acerca de su cognición de los prefijos y sufijos de origen griego o latín, para la construcción de un vocablo en idioma español de importancia en la elaboración de un concepto utilizado en Zoología y en particular en la nomenclatura científico-técnica.

- Entrevista. Para conocer las opiniones de los profesores acerca de la necesidad de construir un diccionario ilustrado en formato digital, de los términos más utilizados en Zoología.

- Consulta a expertos. Se utiliza en el proceso de construcción del diccionario y su diseño inicial, así como para obtener información de credibilidad y concordancia como criterio de valoración de la utilidad del sistema a partir de los indicadores de evaluación elaborados por los autores.

Se esgrimen, además, métodos estadísticos y matemáticos. La muestra seleccionada estuvo constituida por 15 estudiantes que transitaron por el $2^{\text {do }}$ y $3^{\text {er }}$ año de la carrera de Licenciatura en Educación-Biología en la Facultad de Educación Media de la Universidad Central "Marta Abreu" de Las Villas, Villa Clara en la República de Cuba, y 10 profesores que imparten Biología en diferentes universidades del país donde se estudia dicha carrera.

Como se había manifestado con anterioridad, la valoración y estructuración del diccionario se realizó mediante la aplicación del método de criterio de experto, que se sostiene en la consulta a personas que poseen vastos conocimientos del objeto de estudio y es subjetivo.

En la investigación se toma como experto a la persona o grupos de personas capaces de ofrecer, con un máximo de competencia, valoraciones sobre un determinado problema, hacer pronósticos reales y objetivos sobre el efecto, la aplicabilidad, la viabilidad y la relevancia que puede tener en la práctica la propuesta y brindar recomendaciones para perfeccionarla (Armiñana et al., 2020).

A criterio de los autores de esta investigación, los expertos pueden proporcionar valoraciones conclusivas sobre un aspecto explícito, por lo que no obligatoriamente sus apreciaciones tienen que estar destinadas a todo el objeto de estudio. De esta forma la aplicación del método admite alcanzar una concepción de la propuesta más terminada a partir del nivel de valoración de aquellos aspectos de interés para el investigador y de los criterios y sugerencias expresadas por los expertos. En este sentido, es de vital importancia contar con las ideas aportadas y perfeccionar las acciones para su puesta en práctica.

Con la finalidad de agilizar el trabajo de los nueve expertos seleccionados se confeccionó un instrumento, en el cual se refleja una serie de indicadores que permiten valorar la estructura del diccionario. El criterio de evaluación se asume de acuerdo con la escala: Muy Adecuado (MA), Bastante Adecuado (BA), Adecuado (A), Poco Adecuado (PA) e Inadecuado (I).

Para establecer el nivel de competencia de los expertos se manejó el cálculo del coeficiente K (Tabla 1), el cual se establece a partir de la autoevaluación que cada 
aspirante efectúa de su propio nivel de conocimientos en relación al problema a valorar (García \& Fernández, 2008; Mengual, 2011; Zayas, 2011; Armiñana et al., 2020). Como unidad critica se estableció, que aquellos expertos que obtengan una puntuación menor a 0,80 no serán contemplados para el estudio en correspondencia (Cabero \& Llorente, 2014; Armińana et al., 2020).

Tabla 1

Coeficiente de competencia de los expertos que valoraron el diccionario

\begin{tabular}{ccccc}
\hline Expertos & $\begin{array}{c}\text { Coeficiente de } \\
\text { conocimiento }(\mathrm{Kc})\end{array}$ & $\begin{array}{c}\text { Coeficiente de } \\
\text { argumentación }(\mathrm{Ka})\end{array}$ & $\begin{array}{c}\text { Coeficiente de } \\
\text { competencia } \\
(\mathrm{K})\end{array}$ & Valoración \\
\hline 1 & 0,80 & 1,00 & 0,90 & ALTO \\
2 & 0,90 & 1,00 & 0,95 & ALTO \\
3 & 0,90 & 1,00 & 0,95 & ALTO \\
4 & 0,80 & 0,90 & 0,85 & ALTO \\
5 & 0,70 & 0,80 & 0,80 & ALTO \\
6 & 0,70 & 0,80 & 0,85 & ALTO \\
7 & 0,80 & 0,90 & 0,85 & ALTO \\
8 & 0,80 & 0,90 & 0,85 & ALTO \\
9 & 1,00 & 0,80 & 0,90 & ALTO \\
\hline
\end{tabular}

GCI (Kc) Grado de conocimiento e información

Ka Coeficiente de argumentación o fundamentación

$\mathrm{K}$ Coeficiente de competencia

$\mathrm{K}=1 / 2(\mathrm{Kc}+\mathrm{Ka})$

\section{Aspectos éticos}

La investigación estuvo sujeta a normas éticasque posibilitaron promover yasegurar el respeto de todos los participantes en el estudio (estudiantes, profesores y expertos), de modo que se respetaron sus criterios/ opiniones y derechos individuales, para poder generar nuevos conocimientos sin violar los principios éticos de la intimidad y confidencialidad de la información personal, de todos los participantes en la investigación (DHAMM, 2013).

\section{Resultados}

En el diagnóstico preliminar, se examinaron documentos normativos y metodológicos dentro de los que se acentúan los programas de la disciplina Zoología General (Zoología General I y Zoología General II), o sea, Zoología de los invertebrados y vertebrados respectivamente, las orientaciones metodológicas y la bibliografía básica y complementaria, utilizada para el proceso de enseñanza-aprendizaje de la Zoología General en los cursos académicos 20162017 segundo semestre y el primer semestre 2017-2018.

\section{Resultados de la encuesta aplicada a los estudiantes}

La encuesta aplicada a los estudiantes una vez terminado el segundo semestre del $2^{\text {do }}$ año y $1^{\text {er }}$ semestre de $3^{\text {er }}$ ańo de la carrera Licenciatura en Educación-Biología, en los cuales recibieron contenidos concernientes a las asignaturas Zoología de los invertebrados y Zoología de los vertebrados se evidenciaron los siguientes resultados, los cuales se muestran en las Tablas 2 y 3 . Para una mejor comprensión 
de las tablas, se expone a continuación la encuesta aplicada:

\section{ENCUESTA}

ESTUDIANTE

A continuación, se le ofrece un instrumento que permitirá determinar aspectos importantes para perfeccionar un trabajo investigativo que se está llevando a cabo en la Facultad de Educación Media y en particular en el departamento de Ciencias Naturales. Disculpe las molestias que esto le pueda ocasionar. En ningún caso aparecerá el nombre de algunos de ustedes, por lo que no deben preocuparse por ello.

Muchas gracias

Objetivo: Determinar la cognición de los prefijos y sufijos de origen griego o latín, para la construcción de un vocablo en idioma español de importancia en la elaboración de un concepto utilizado en Zoología y en particular de la nomenclatura científico - técnica.

\section{CUESTIONARIO}

1. Elabore algún término utilizado en la disciplina Zoología con los prefijos que le mostramos a continuación.

\section{Blasto- Cromo- a- Foro- Fago- \\ Para- Ecto- Uro- Iso- Morfo-}

2. Elabore otros términos usado en la disciplina Zoología, pero ahora utilice los sufijos.

-blasto, -cromo, -ploide, -foro, -fago, -podo, -ptero, -uro, -zoo, -morfo.

Leyenda: $\left(^{*}\right)$ elaboró el término.

Tabla 2

Resultado de la formación de términos con prefijos. Curso 2016-2017, segundo semestre del segundo año y primer semestre del 3er año del curso 2017-2018.

\begin{tabular}{|c|c|c|c|c|c|c|c|c|c|c|c|}
\hline Est. & Blasto- & Cromo- & $a-$ & ana- & Fago- & Para- & Ecto- & Uro- & Iso- & Morfo- & $(\%)$ \\
\hline 1 & & * & $*$ & & & * & & * & & * & 50 \\
\hline 2 & & & $*$ & & * & & & & & * & 30 \\
\hline 3 & & * & & & $*$ & & $*$ & & $*$ & $*$ & 50 \\
\hline 4 & & & & & & * & * & & & * & 30 \\
\hline 5 & * & * & & * & * & & * & * & & * & 70 \\
\hline 6 & & & & & & * & & & & * & 20 \\
\hline 7 & & * & * & & & & & * & & * & 40 \\
\hline 8 & * & & & & & & * & & & * & 30 \\
\hline 9 & & & * & & & * & * & & & * & 40 \\
\hline 10 & & * & & * & & & & & & * & 30 \\
\hline 11 & * & & * & $*$ & * & & & * & $*$ & * & 70 \\
\hline 12 & & * & & * & * & & & & & * & 40 \\
\hline 13 & * & & * & & & & * & & & * & 40 \\
\hline 14 & & * & $*$ & & & * & * & & & * & 50 \\
\hline 15 & & * & & * & & & * & & & * & 40 \\
\hline Total & 4 & 8 & 7 & 5 & 5 & 6 & 8 & 4 & 2 & 15 & - \\
\hline $\begin{array}{l}\text { Los } \\
\text { estudia } \\
\text { blastop }\end{array}$ & $\begin{array}{l}\text { términ } \\
\text { tes } \\
\text { ro, by }\end{array}$ & $\begin{array}{l}\text { elab } \\
\text { ron } \\
\text { stóme }\end{array}$ & & $\begin{array}{r}\text { por } \\
\text { siguie } \\
\text { omos }\end{array}$ & $\begin{array}{l}\text { los } \\
\text { tes: }\end{array}$ & $\begin{array}{l}\text { pat } \\
\text { ect } \\
\text { mo }\end{array}$ & $\begin{array}{l}\text { oos, } \\
\text { ermo } \\
\text { ología }\end{array}$ & $\begin{array}{l}\text { para } \\
\text { mor }\end{array}$ & netría & $\begin{array}{l}\text { ectc } \\
\text { los, } \\
\text { morfol }\end{array}$ & م. \\
\hline
\end{tabular}

agnato, anuro, anatomía, fagocitosis, 
Tabla 3

Resultado de la formación de términos con sufijos. Curso 2016-2017, segundo semestre del segundo año y primer semestre del 3er año del curso 2017-2018.

\begin{tabular}{cccccccccccc}
\hline Est. & -blasto & -cito & -ploide & -foro & -podo & -gnato & -ptero & -uro & -zoo & -morfo & $(\%)$ \\
\hline 1 & & $*$ & $*$ & $*$ & & & $*$ & & $*$ & & 50 \\
2 & & $*$ & $*$ & & $*$ & & $*$ & $*$ & $*$ & 60 \\
3 & & $*$ & & & $*$ & & & & $*$ & 30 \\
4 & & & & & & & $*$ & & $*$ & & 20 \\
5 & $*$ & & $*$ & & $*$ & $*$ & $*$ & $*$ & $*$ & $*$ & 80 \\
6 & & & & & & & $*$ & & $*$ & & 20 \\
7 & & $*$ & & $*$ & $*$ & & & & $*$ & & 40 \\
8 & & $*$ & & $*$ & & & & & $*$ & \\
9 & & & & $*$ & & & $*$ & & $*$ & & 30 \\
10 & & & & & & & & $*$ & $*$ & & 20 \\
11 & $*$ & & $*$ & & $*$ & $*$ & $*$ & $*$ & $*$ & & 70 \\
12 & & $*$ & & $*$ & & & & & $*$ & & 30 \\
13 & & & $*$ & & & & & & $*$ & & 20 \\
14 & $*$ & & & & $*$ & & $*$ & & $*$ & & 40 \\
15 & & & $*$ & $*$ & & & & & $*$ & $*$ & 40 \\
\hline Total & 3 & 6 & 5 & 6 & 6 & 2 & 7 & 3 & 15 & 2 & \\
\hline
\end{tabular}

Nota: $\left.{ }^{*}\right)$ elaboró el término.

Los otros términos elaborados, pero utilizando los sufijos fueron los siguientes: diplópodos, porocito, haploide, diploide, poliplacóforo, agnato, diptero, coleóptero, anuro, protozoo, hidrozoo, y amorfo.

Con relación a la entrevista realizada a los 10 profesores de diferentes universidades del país que han impartido Zoología develó los siguientes resultados:

El 100\% de los entrevistados no posee ningún diccionario que aborde términos que se utilicen en Zoología, que incluya las raíces del origen del término ya sea en latín o griego.

Acerca de las habilidades intelectuales que poseen los estudiantes respecto al origen de las palabras en latín o griego de los diferentes términos que se utilizan en zoología, el $100 \%$ de los profesores manifiestan que sus estudiantes poseen insuficiencias en el significado de muchos términos, fundamentalmente de índole taxonómico.

El 100\% manifiestan que sería de vital ayuda la elaboración de un diccionario, que contribuya al desarrollo de habilidades intelectuales en los estudiantes, relacionado con la búsqueda de términos cuyo origen es el latín o el griego.

En tal sentido, la experiencia de los autores, apoyada en la observación sistemática del proceso de enseñanza - aprendizaje de la Zoología de los invertebrados y vertebrados durante dos cursos, la revisión de documentos y el análisis de los instrumentos aplicados, permitieron determinar las carencias, para perfeccionar el proceso de enseñanza aprendizaje de la Zoología. 


\section{Carencias}

- Insuficiente conocimiento por parte de los estudiantes del latín o el griego, para poder interpretar el significado en idioma español de diferentes términos utilizados con mayor frecuencia en Zoología.

- Escasa cognición de los prefijos y sufijos de origen griego o latín, para la construcción de un vocablo en idioma español de importancia en la elaboración de un concepto utilizado en Zoología $y$ en particular en la nomenclatura científico - técnica, aunque las mayores carencias se observan en la utilización de los sufijos.

- Insuficientes conocimientos sobre el origen de un determinado término utilizado en zoología y posterior elaboración de un concepto.

- Inexistencia de un glosario de términos en la literatura básica y complementaria para el estudio de la Zoología.

\section{Potencialidades}

- Motivación por parte de los profesores por hacer del proceso de enseñanzaaprendizaje de la Zoología, más actualizado y dinámico en concordancia con las transformaciones que se ejecutan en la enseñanza superior en Cuba.

- Interés por elaborar un medio de enseñanza en formato digital, en este caso un diccionario de términos más utilizados en Zoología, para minimizar las carencias presentadas por los estudiantes.

Estas regularidades conducen a la necesidad de elaborar en un periodo de dos ańos el "Diccionario ilustrado de algunos términos utilizados en Zoología”.

Construido el diccionario en su primera versión, se les entregó a los expertos para que realizaran las valoraciones pertinentes, cuyos resultados se exponen en la Tabla 4.

Tabla 4

Valoración por criterios de expertos de la propuesta del "Diccionario ilustrado de algunos términos utilizados en Zoologia".

\begin{tabular}{|c|c|c|c|}
\hline INDICADORES PROPUESTOS & MA (\%) & $\mathrm{BA}(\%)$ & A (\%) \\
\hline $\begin{array}{l}\text { 1. Estructuración del diccionario que se propone, según las nor- } \\
\text { mas internacionales. }\end{array}$ & $9100)$ & & \\
\hline $\begin{array}{l}\text { 2. Correspondencia de los términos y conceptos científicos, con } \\
\text { el nivel de desarrollo de la Zoología en el mundo y en Cuba } \\
\text { en los momentos actuales. }\end{array}$ & $8(89)$ & & \\
\hline $\begin{array}{l}\text { 3. Correspondencia del vocabulario técnico que se observa en el } \\
\text { diccionario en correspondencia con las particularidades sicope- } \\
\text { dagógicas de los estudiantes al cual va dirigido. }\end{array}$ & $9(100)$ & & \\
\hline 4. Calidad de las imágenes en la iconoestructura del diccionario. & $9(100)$ & & \\
\hline $\begin{array}{l}\text { 5. Contribución de las imágenes seleccionadas a la comprensión } \\
\text { de algunos términos relacionados con el estudio de la Zoología } \\
\text { de los invertebrados y cordados. }\end{array}$ & $9(100)$ & & \\
\hline $\begin{array}{l}\text { 6. Contribución del diccionario a la motivación de los estudian- } \\
\text { tes por el estudio la Zoología. }\end{array}$ & $8(93)$ & & \\
\hline $\begin{array}{l}\text { 7. Contribución del diccionario a la autogestión de los estudian- } \\
\text { tes de sus propios conocimientos. }\end{array}$ & $9(100)$ & & \\
\hline $\begin{array}{l}\text { 8. Contribución del diccionario al desarrollo de habilidades in- } \\
\text { telectuales. }\end{array}$ & $8(89)$ & & \\
\hline $\begin{array}{l}\text { 9. Pertinencia del diccionario elaborado, como vía para interpre- } \\
\text { tar términos que provienen del latín o el griego. }\end{array}$ & $9(100)$ & & \\
\hline
\end{tabular}


En correspondencia con las diferentes ideas y criterios sobre las bondades, deficiencias e insuficiencias que presenta el diccionario en su concepción teórica y que pudiera presentar al ser aplicado en la práctica, con el fin de poder generar un perfeccionamiento de este, los expertos abordaron algunos aspectos, los cuales se expresan a continuación textualmente y que fueron objeto de análisis por los investigadores. Algunas de las observaciones fueron las siguientes:

"Considero que para que la obra adquiera mucha mayor relevancia se debería incluir más términos y hacer corresponder los términos seleccionados con los que aparecen en el libro de texto de Biología 2 de $8^{\text {vo }}$ grado".

"Introducir en el diccionario un prólogo elaborado por algún investigador ajeno a los autores del mismo"

A continuación, se exponen algunos de los criterios emitidos por los expertos, a favor del "Diccionario ilustrado de algunos términos utilizados en Zoología".

Experto 3: "Durante 30 años, he estado impartiendo las asignaturas Zoología de los invertebrados y vertebrados, la cual ha recibido algunos otros nombres, en los diferentes planes de estudio desde el plan A, implementado en el curso 1976-1977 hasta el plan E actual, y no había tenido la oportunidad de tener en mis manos un diccionario ilustrado en formato electrónico, donde se plasmaran diferentes términos, relacionados con el estudio de los animales y que incluyera las raíces de procedencia del término, ya sea en griego o en latín. A mi juicio este diccionario que se me presenta para valorarlo, es una obra de alto rigor científico, que permitirá a los estudiantes de la Licenciatura en Educación-Biología conocer el origen de diferentes términos y su significado y permitirá comprender además el significado de numerosos nombres científicos de especies de no cordados y vertebrados".

"Quisiera añadir que en el proceso de enseñanza-aprendizaje, en lo que a la formación de conceptos se refiere; un glosario de términos, un vocabulario técnico o un diccionario propiamente dicho, adquiere una importante función, porque permite hacer una retroalimentación al estudiante en el momento en que este lo necesite, facilita la profundización en la asignatura de forma independiente, permite la comparación de conceptos dados por distintos autores con respecto a un mismo término y facilita abordar la literatura especializada".

Experto 9: "La propuesta del diccionario ilustrado de algunos términos utilizados en Zoología, a mi juicio posibilitará y permitirá el desarrollo de estrategias más dinámicas de aprendizaje, ya que los estudiantes podrán interactuar con él, seleccionando aquellos términos que están en griego o latín que les permitan entender con mayor precisión un concepto zoológico determinado en español".

"Me ha gustado mucho la selección de los términos que se encuentran en el diccionario y las fotos que tienen una gran calidad, es un diccionario muy atractivo y creo que contribuirá en los estudiantes a motivarse por las asignaturas relacionadas con el estudio de los animales. Considero que se puede seguir trabajando en la búsqueda de otros términos para incluirlos en él”

Experto 10: "Considero muy novedosa la propuesta, al revisarlo me invita a la búsqueda de términos, porque posee un agradable diseño, a mi juicio en todos los sentidos, excelentes fotos, que ayudan al entendimiento de la terminología que aparecen en el mismo".

"Muy acertado por parte de los autores del 
capítulo 1, donde se dedicada a los prefijos y sufijos más comunes utilizados en la zoología, porque esto permite comprender muchos términos por parte de los estudiantes y profesores".

"El diccionario posee una gran vistosidad en cuanto a la calidad y variedad de las imágenes y del diseño gráfico".

El examen de los resultados logrados para los indicadores propuestos, y al mismo tiempo con el análisis de las opiniones adicionalmente formuladas por los expertos, constituyó un momento de meditación para la ejecución de modificaciones.

Al considerar los resultados obtenidos y las proposiciones realizadas por los expertos se realizaron los ajustes pertinentes, que a continuación se exponen:

- Se hizo coincidir los términos seleccionados para el diccionario, con el glosario de términos que se observa en el libro de Biología 2 de 8 vo grado, donde se estudian los animales.

- Se le realizó el prólogo al diccionario.

\section{Propuesta de solución al problema científico y objetivo de la investigación}

Una vez realizada la determinación de necesidades, utilizando diferentes encuestas, entrevistas y otros métodos empíricos. Analizadas las potencialidades y carencias y ser sometido a criterios de expertos la primera versión de la obra, se elaboró de manera definitiva el "Diccionario ilustrado de algunos términos utilizados en Zoología”.

La obra, considerada dentro de la perspectiva de la estilística funcional, se presenta como un texto de estilo científico, y aprovechando las posibilidades que ofrece la lingüística y las Tecnologías de la Información y la Comunicación. En él quedan evidenciados los rasgos distintivos del género científico, porque se definen diferentes términos utilizados en Zoología con el rigor establecido para este tipo de obra.

La selección de los términos que se han incluido en este diccionario, va más allá de lo que se pudiera entender como zoológicos propiamente dicho, partiendo de la opinión de que todos coadyuvan al entendimiento cabal de la Zoología. No obstante, pudiera darse el caso de que algunos términos o conceptos no estén presentes en este diccionario por diversas razones; como, por ejemplo: la falta de definición del concepto en las literaturas consultadas, que no se recordara para su inclusión, o simplemente, que se consideró no incluir tal o más cual, porque no era necesario; incluso, se obviaron muchos términos de índole taxonómico.

Se hace necesario recordar, que la mayor parte de los conceptos utilizados en zoología tienen sus raíces en el griego o el latín, que son lenguas romances, terminología que, desde el punto de vista de su significado, puede ser desconocida por los profesores o por los estudiantes. Es por ello que se decidió incluir en esta obra una primera sección dedicada a los "prefijos y sufijos más comunes utilizados en la Zoología”.

Para una mejor comprensión de lo abordado anteriormente, se muestran los siguientes ejemplos. La palabra HIPO, como prefijo significa por debajo, así el enanismo es producido por una hipofunción de la glándula hipófisis; hipocóndrico, que, en anatomía humana, significa región situada debajo del gran cartílago que une a las costillas falsas; hipogástrico, todo lo que está por debajo del estómago. Sin embargo; HIPO como concepto significa, entrada violenta del aire en el tórax debida a una rápida contracción del diafragma. 
La segunda sección o capítulo está dedicada al vocabulario técnico general y en la mayor parte de ella, en que no aparece la etimología de una palabra determinada, se indican sus componentes o partes de ellos y la etimología en la sección primera; como, por ejemplo, ACTINOPTERYGII. Las partes de este vocablo son: ACTINO y PTERYGII, esto significa «radio» $\mathrm{y}$ «aleta» respectivamente. De aquí que toda la palabra signifique de aletas radiadas. En el caso de los términos, SARCOPTERYGII o CROSSOPTERYGII, solo se daría la etimología del prefijo en griego o latín, porque el sufijo pterygii, ya se había definido antes, esto puede suceder también con los prefijos. En fin, un gran número de nombres técnicos pueden traducirse a sus equivalentes castellanos, separando las palabras en partes.

El diccionario que se presenta se clasifica en especializado, porque está dedicado a palabras o términos que pertenecen a un campo o técnica determinados como, es el caso de la Biología, proporciona información sobre el significado de tales palabras o términos.

Es preciso puntualizar, que en el diccionario se ofrece además, la etimología de las palabras que según el DRAE (2012), proviene del latín etymología y éste del griego étv $\mu о \lambda o \gamma i \alpha$,

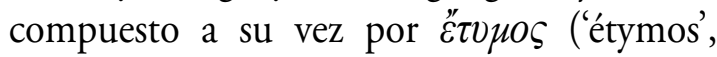

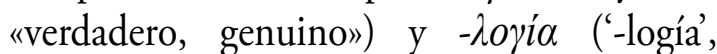
«tratado, estudio»), al estudio del origen de las palabras, cuándo son incorporadas a un idioma, de qué fuente, y cómo su forma y significado han cambiado.

Según Armiñana (2019), la etimología es una disciplina relacionada con la filología y la lingüística histórica que estudia el origen de las palabras investigando su significación originaria y su forma, así como los posibles cambios sufridos a lo largo del tiempo.
Para los autores de esta investigación, la etimología ayuda a entender el significado de las palabras, a ampliar el vocabulario y corregir la ortografía.

El diccionario consta con un total de 125 páginas y está estructurado según las normas internacionales, su macroestructura, microestructura, hiperestructura e iconoestructura, están muy bien definidas y es de subrayar que con relación a la iconoestructura se ha sido muy riguroso en la selección de las 389 fotos que conforman la obra, y todas ellas a juicio de los autores y criterios de expertos sirven de referente, para entender mejor el significado de diferentes términos.

La Figura 1 muestra la portada del diccionario. A continuación, se exponen la página 7 donde comienza el capítulo I (Figura 2) y la página 78 del capítulo II (Figura 3) para que el lector tenga una noción mucho más exacta acerca del diccionario elaborado.

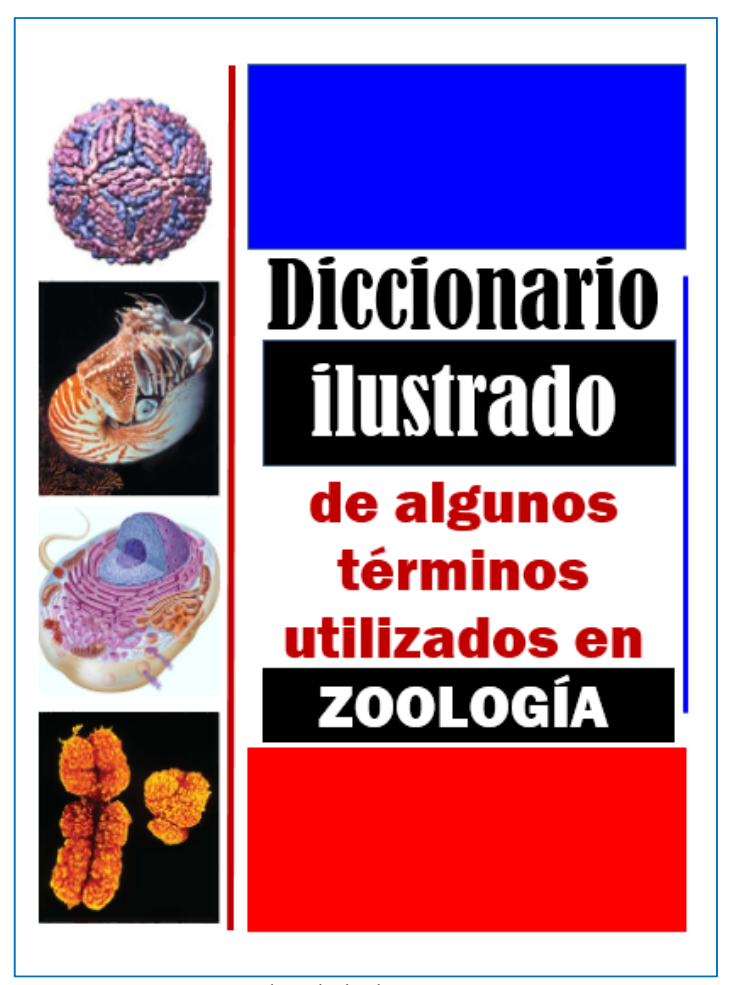

Figura 7. Portada del diccionario 


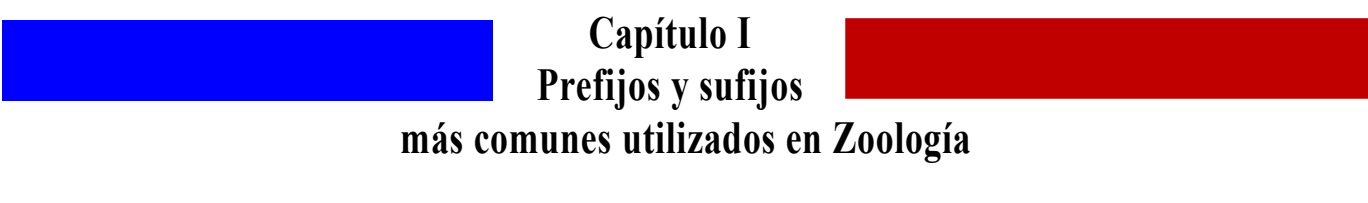

a- (griego, no): es un sufijo negativo: como, por ejemplo., acelo, sin celoma, acéfalo, sin cabeza.

ab- (latín, de, desde): opuesto a ad, hacia c.p.e, aboral opuesto a la boca; abductor, músculo que separa de una posición dada. acanthia (Gr. akantia, espina).

acro- (Gr. akros, extremo): c.p.e, acrodonto, acromegalia, acrosoma.

actino- (Gr. aktis, radio): c.p.e actinópodo, pie en forma de radio, actinotroca, larva con rueda, o corona radiada.

ad-(L. hacia): opuesto a ab, c.p.e, aductor, músculo que lleva a una porción dada.

aferente- (L. ad + ferre, llevar): que lleva a una posición dada opuesto a eferente, c.p.e nervio aferente, vaso sanguíneo aferente.

an- (Gr. no): como a, usado ante vocal $\mathbf{o}$ la h, c.p.e anamniota, anhídrido.

ana-. (Gr. por, a través): c.p.e, analogía, semejanza; análisis, separación; anatomía, corte.

andro-(Gr. aner, varón): c.p.e., andrógeno, hormona, masculina.

anfi- (Gr. amphi, alrededor, a los lados): c.p.e., Amphioxus, puntiagudo por ambos extremos; anfipodo, que posee patas torácicas y abdominales.

anto- (Gr. anthos, flor): c.p.e, antozoos, Celenterados parecidos a flor.

antropo- (Gr. antropus, hombre): c.p.e., antropoide, mono semejante al hombre.

apical-(L. apex, punta, extremidad): perteneciente a una punta o que está en ella o cerca de ella-.

apo-(Gr. de, desde): compárese con L. ab; c.p.e, apópilo, abertura muy separada, en las esponjas es un orificio del canal radial hacia el espongiocele.

-apsido (Gr. apsis, bóveda): c.p.e., diápsido que tiene dos bóvedas en el cráneo.

arqu-, arqueo, arqui- (Gr. archaios, antiguo, primitivo): c.p.e., arquenteron, primer intestino embrionario; arqueanélido, anélido primitivo.

artro- (Gr. artrhon, articulación): c.p.e., artrópodo, con patas articuladas, artritis, infamación de las articulaciones.

asc-, asco (Gr. ascos, saco): c.p.e., aschelminthes, gusanos en forma de saco, ascogonium, estructura en forma de sac

Figura 2. Capítulo I. Prefijos y sufijos más comunes utilizados en Zoología. 


\section{Capítulo II}

\section{decimotercera letra y la décima consonante del alfabeto español y del alfabeto latino básico. Su nombre en español es femenino: la eme, en plural emes.}

Macrófago m: que recolecta e ingiere grandes partículas alimentarias.

Macruros, macrura (Gr. grande + oura, cola): especies de crustáceos que poseen el abdomen largo; c.p.e., los camarones, langostas, esquilas, y otros.

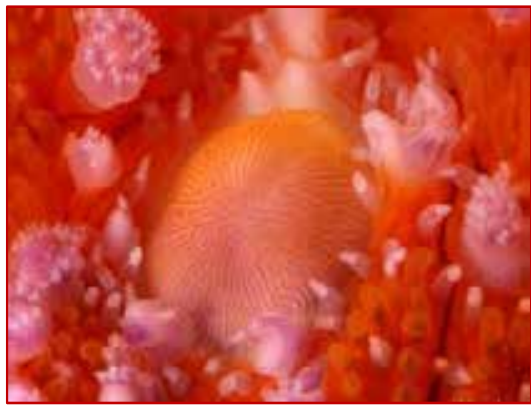

Madreporito (L. mater, madre + Gr. poros, poro + ite, naturaleza de.): estructura de tamiz que se encuentra en la entrada del sistema acuífero ambulacral, en ciertos equinodermos, c.p.e., en erizo de mar.

Malacostracos, malacostraca (Gr. malakos, blando + ostrakon, concha): incluye los crustáceos grandes y otros de menor de tamaño, c.p.e., cangrejos, cochinillas de humedad, galeras, y otros.

Malófagos, mallophaga (Gr. mallos, lana + phago, comer): orden de los insectos donde se ubican los piojos mordedores, ectoparásitos de las aves y de algunos mamíferos, se alimentan de fragmentos de piel, pluma o pelo.

Mamíferos, mammalia (L. mamma, mama, pecho): término definido por Linneo, (1758) al grupo de animales que son nutridos con leche
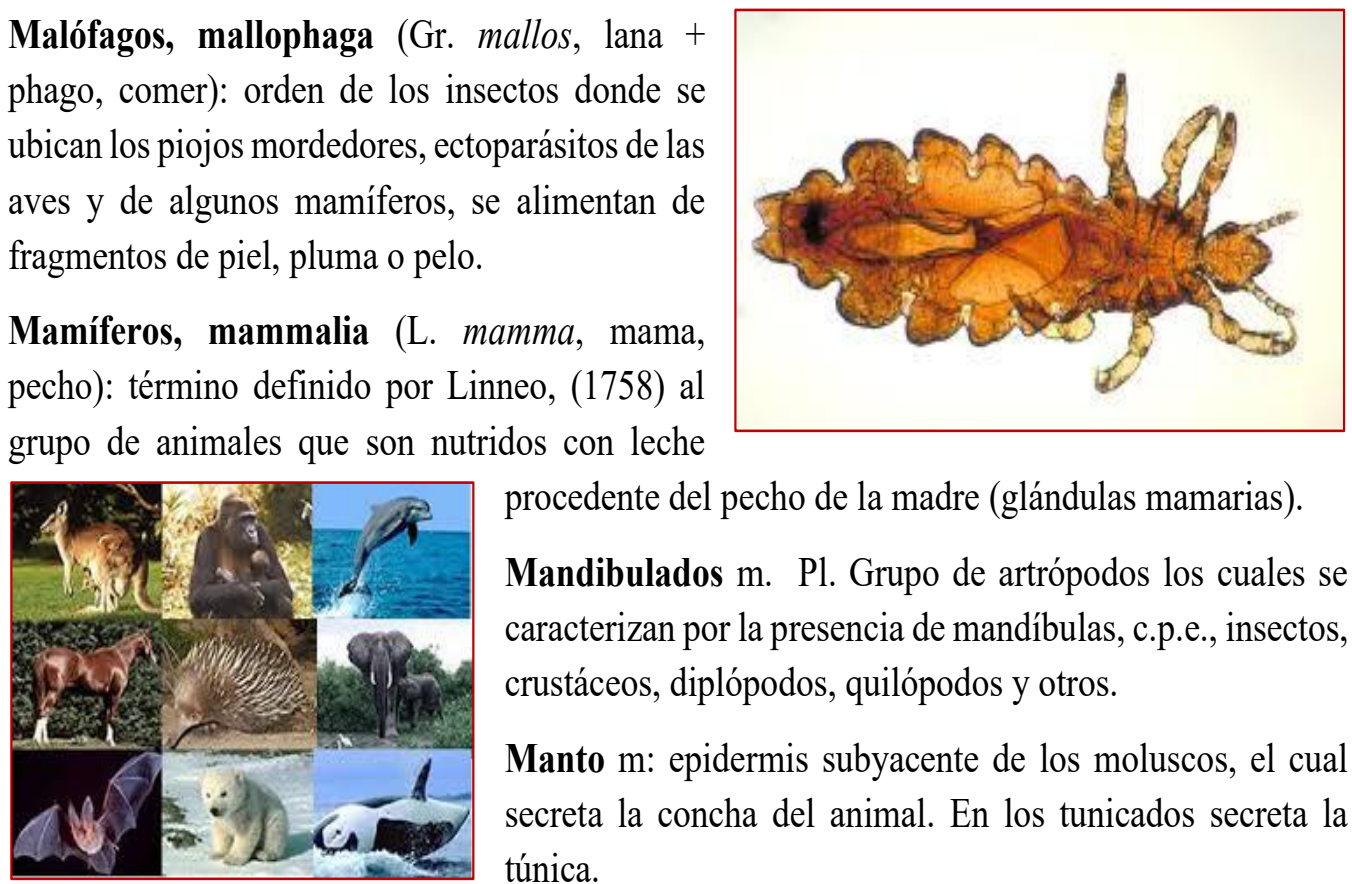

procedente del pecho de la madre (glándulas mamarias).

Mandibulados m. Pl. Grupo de artrópodos los cuales se caracterizan por la presencia de mandíbulas, c.p.e., insectos, crustáceos, diplópodos, quilópodos y otros.

Manto m: epidermis subyacente de los moluscos, el cual secreta la concha del animal. En los tunicados secreta la túnica.

Figura 3. Capítulo II. M decimotercera letra y la décima consonante del alfabeto español y del alfabeto latino básico 
Una vez elaborado, definitivamente, el diccionario, se implementa en los cursos 2018-2019 segundo semestre del segundo año y primer semestre del 3er año del curso 2019-2020.

Culminado el primer semestre del $3^{\text {er }}$ año, una vez concluida la impartición de las asignaturas Zoología de los invertebrados y vertebrados respectivamente se aplica la misma encuesta a una muestra de 15 estudiantes del curso 2019 - 2020. Las Tablas 5 y 6 muestran los resultados obtenidos.

Tabla 5

Resultado de la formación de términos con prefijos una, vez implementado el diccionario. Curso 2018-2019-Segundo semestre del segundo año y primer semestre del $3^{\text {er }}$ año del curso 2019-2020.

\begin{tabular}{cccccccccccc}
\hline Est. & Blasto- & Cromo- & a- & Foro- & Fago- & Para- & Ecto- & Uro- & Iso- & Morfo- & $(\%)$ \\
\hline 1 & $*$ & $*$ & $*$ & & $*$ & $*$ & $*$ & $*$ & $*$ & $*$ & 90 \\
2 & $*$ & $*$ & $*$ & $*$ & $*$ & $*$ & $*$ & $*$ & $*$ & $*$ & 100 \\
3 & $*$ & $*$ & $*$ & $*$ & $*$ & $*$ & $*$ & $*$ & & $*$ & 90 \\
4 & $*$ & $*$ & $*$ & & $*$ & $*$ & $*$ & $*$ & & $*$ & 80 \\
5 & $*$ & $*$ & & & $*$ & & $*$ & $*$ & $*$ & $*$ & 70 \\
6 & $*$ & $*$ & $*$ & & & & $*$ & & & $*$ & 50 \\
7 & $*$ & $*$ & $*$ & & $*$ & $*$ & $*$ & & $*$ & $*$ & 80 \\
8 & $*$ & $*$ & & $*$ & $*$ & $*$ & $*$ & $*$ & $*$ & $*$ & 90 \\
9 & $*$ & $*$ & $*$ & & $*$ & & $*$ & $*$ & $*$ & $*$ & 80 \\
10 & $*$ & $*$ & $*$ & $*$ & $*$ & & & $*$ & $*$ & $*$ & 80 \\
11 & $*$ & $*$ & $*$ & $*$ & $*$ & $*$ & $*$ & $*$ & & $*$ & 90 \\
12 & $*$ & $*$ & & $*$ & $*$ & $*$ & $*$ & & & $*$ & 70 \\
13 & $*$ & $*$ & & $*$ & $*$ & & $*$ & & $*$ & $*$ & 70 \\
14 & $*$ & $*$ & $*$ & & & & $*$ & & $*$ & $*$ & 60 \\
15 & $*$ & $*$ & & & $*$ & $*$ & & $*$ & $*$ & $*$ & 70 \\
Total & 15 & 15 & 10 & 7 & 13 & 9 & 13 & 10 & 10 & 15 & \\
\hline
\end{tabular}

Nota: $(\mathrm{X})$ elaboró el término

Los términos elaborados por los estudiantes fueron los siguientes: blastoporo, blastómero, blastocele, blastodisco, cromosoma, cromatóforo, cromocito, cromatina, cromátida, agnato, anuro, anatomía, fagocitosis, fagocitar, fago, parazoos, parasimpático, parapléjico, ectoparásito, ectodermo, urocordados, urodelos, isóptero, isópodo, morfología, morfometría, morfológico.

Nota: (X) elaboró el término

Los términos elaborados por los estudiantes fueron los siguientes: diploblásticos, triploblásticos, osteoblasto, escleroblasto, espongioblasto, citocromo, artiodáctilo, perisodáctilo, poliplacóforo, monoplacóforo, macrófago, necrófago, micrófago, agnato, quetognato, coleóptero, lepidóptero, diptero, anuro, anomuro, blastómero, macrómero.

Como se puede apreciar, los resultados obtenidos por los estudiantes una vez implementado el diccionario es muy superior, independientemente de que la muestra no es la misma, pero se intencionó la selección investigando en el diagnóstico, los estudiantes con niveles de asimilación similares a la de la muestra escogida en los cursos 2016 - 2017 y 2017 - 2018 . 
Tabla 6

Resultado de la formación de términos con los sufijos, una vez implementado el diccionario. Curso 2018-2019 segundo semestre del segundo año y primer semestre del 3er año del curso 2019-2020.

\begin{tabular}{cccccccccccc}
\hline Est. & -blasto & -cromo & -dáctilo & -foro & -fago & -gnato & -ptero & -uro & -mero & -morfo & $(\%)$ \\
\hline 1 & $*$ & $*$ & $*$ & $*$ & & $*$ & $*$ & & & $*$ & 60 \\
2 & $*$ & & $*$ & $*$ & $*$ & $*$ & $*$ & $*$ & $*$ & & 80 \\
3 & $*$ & $*$ & $*$ & $*$ & $*$ & & $*$ & $*$ & & & 70 \\
4 & $*$ & $*$ & & $*$ & & $*$ & $*$ & $*$ & & & 60 \\
5 & $*$ & & $*$ & $*$ & & & $*$ & $*$ & $*$ & & 60 \\
6 & $*$ & $*$ & $*$ & $*$ & $*$ & & $*$ & $*$ & & $*$ & 80 \\
7 & $*$ & $*$ & & $*$ & & $*$ & $*$ & $*$ & & $*$ & 60 \\
8 & $*$ & & $*$ & & $*$ & $*$ & $*$ & $*$ & & & 60 \\
9 & $*$ & $*$ & $*$ & $*$ & & $*$ & $*$ & & $*$ & & 70 \\
10 & $*$ & $*$ & $*$ & $*$ & $*$ & & $*$ & $*$ & $*$ & $*$ & 90 \\
11 & $*$ & $*$ & $*$ & & $*$ & & $*$ & $*$ & & $*$ & 70 \\
12 & $*$ & $*$ & $*$ & $*$ & $*$ & & $*$ & $*$ & & $*$ & 80 \\
13 & $*$ & & & $*$ & $*$ & $*$ & $*$ & & $*$ & & 60 \\
14 & $*$ & $*$ & $*$ & $*$ & & $*$ & $*$ & & $*$ & $*$ & 80 \\
15 & $*$ & & $*$ & $*$ & $*$ & $*$ & $*$ & $*$ & $*$ & & 80 \\
Total & 15 & 10 & 12 & 13 & 9 & 9 & 15 & 11 & 7 & 7 & \\
\hline
\end{tabular}

En tal sentido, la efectividad del diccionario es incuestionable, los estudiantes pudieron formar muchos más términos utilizando los prefijos y sufijos. Sin embargo, es preciso destacar que la orientación eficaz hacia el estudio independiente por parte del profesor y las diferentes actividades realizadas durante el proceso de enseńanza-aprendizaje de la Zoología, encaminadas hacía la búsqueda de las raíces griegas o latinas de diferentes términos, posibilitó estos resultados.

\section{Discusión}

En primer lugar, se desea plasmar que el diccionario, elaborado para el proceso de enseñanza aprendizaje de la Zoología, se sustenta en la Teoría Histórico Cultural de Vygotsky, al considerar el papel del profesor, del estudiante y del grupo como mediadores socioculturales que promueven el desarrollo, tiene en cuenta la atención a la diversidad y al autoaprendizaje de los estudiantes, así como los requerimientos didácticos para su utilización.

Debido a que el diccionario introduce muchos términos que proviene del latín o el griego fundamentalmente, algunos investigadores sostienen que estas lenguas son muertas. Sin embargo, los autores de esta investigación no concuerdan con tal afirmación sí se tiene en cuenta que determinadas ciencias todavía utilizan una gran cantidad de su léxico y de que existen aún muchas personas que son capaces de hablarlas como segunda lengua. Estas lenguas no han muerto, nunca murieron y no morirán, siguen vivas en nuestra lengua materna, el español y además en nuestra cultura. Sin el latín no existiría el castellano, ni las demás lenguas románicas, también conocidas como lenguas romances o neolatinas, ni un vocabulario científico técnico. 
Es válido puntualizar que cualquier ciencia es ciencia, solo en la medida en que establezca una estrecha relación entre un sistema de conocimientos y conceptos con los métodos del conocimiento mismo y la práctica social. Por su parte, una asignatura es el reflejo docente de una ciencia; determina los momentos más importantes de la ciencia en cuestión, su eje de conceptos y métodos son llevados al aula. Consecuentemente en la asignatura conceptos y métodos se llevan de la mano. La eficiencia de un programa podría medirse precisamente por la estrecha vinculación entre los conceptos y los métodos.

Así el profesor debe establecer relación de métodos, conceptos y prácticas, en sus aspectos más fundamentales. El diccionario elaborado resuelve uno de estos aspectos y coadyuva en el entendimiento de los otros en la didáctica.
La construcción del diccionario no resultó una tarea fácil, porque se tuvo que extraer cada vocablo o término de la documentación revisada (Collins et al., 2016; Vlachos, 2019; Fellowes, 2020), y tratar de definir su significado preciso. Agotar la denotación de una palabra y todas sus connotaciones. Indagar de dónde proviene, qué otras palabras la sucedieron. Enumerar de cuántas expresiones forman parte y buscar ejemplos claros y divulgativos de su empleo.

En la elaboración del diccionario, se ha respetado los principios didácticos para la producción de la literatura docente (Zumbado, 2004; Armiñana, 2014a) y las funciones didácticas (Zuev, 1987; Zumbado, 2004; Armiñana, 2015b, 2016c).

\section{Referencias}

Acosta, L.E. (2007). Zoological Nomenclature: opportunities and challenges in the digital age. Nomenclatura Zoológica: oportunidades y desafíos en la era digital. Revista de la Sociedad Entomológica Argentina, 66, 27-40.

Ameyaw, Y. \& Kyere, I. (2019). Mapping Biological Concepts: Concept-Vee maps an improver of students' performance in photosynthesis. International Journal of Innovative Science Engineering and Technology, 6: 169-181.

Armiñana, G.R. (2014). Sistema de Medios de Enseñanza Asistido por Computadoras, para el perfeccionamiento del proceso de enseñanza-aprendizaje de la Zoología General I. Tesis presentada en opción al grado científico de Doctor en Ciencias Pedagógicas. Universidad de Ciencias Pedagógicas "Félix Varela Morales". Villa Clara. Cuba. 215 pp.

Armiñana, G.R. (2015). Apuntes para la elaboración de un Libro de Texto. Revista del Instituto Pedagógico Latinoamericano y Caribeño. 11 pp.

Armiñana, G.R. (2016a). Programa de la asignatura Zoología General I. Universidad Central «Marta Abreu» de Las Villas. Villa Clara, Cuba. 15 pp.

Armiñana, G.R. (2016b). Programa de la asignatura Zoología General II. Uni- 
Rafael Arminaña-García - Rigoberto Fimia-Duarte - José Iannacone - Lorena Alvariño YANIRA ZAITA-FERRER

versidad Central «Marta Abreu» de Las Villas. Villa Clara, Cuba. 12 pp.

Armiñana, G.R. (2016c). El libro de texto como medio de enseñanza para las Ciencias en la Educación Superior. En: CD. Compilación de experiencias en la elevación de la calidad de la Educación Cientifica en la provincia de Villa Clara. Órgano Editor Educación Cubana. 15 pp.

Armiñana, G.R. (2019). Diccionario ilustrado e interactivo, una vía novedosa para el proceso de enseñanza - aprendizaje de la Zoología. Congreso Internacional Pedagogía 2019. Universidad Central «Marta Abreu» de las Villas, Villa Clara, Cuba. 13 pp.

Armiñana, G.R., Castillo, F.Y., Mesa, C.N., Fimia, D.R., Leyva, H.J., Iannacone, J., Durán, F.Y. \& Fábrega, O.G. (2020). Nueva concepción didáctica para el proceso de enseñanza - aprendizaje de la zoología de los cordados. Paideia XXI, 10, 33-57.

Armiñana, G.R., Garcés, F.J., Castillo, F.Y., Fimia, D.R., Guerra, V.Y. \& Iannacone, J. (2020). Los mapas conceptuales en el proceso de enseñanza-aprendizaje de la Biología 2. Paideia XXI, 10, 59-75.

Banasco, A.J., Armiñana, G.R. \& Garcés, F.J. (2016). Programa de la disciplina Zoología General. Ministerio de Educación Superior. La Habana, Cuba. 11 pp.

Cabero, A.J. \& Llorente, C.M.C. (2013). La aplicación del juicio de experto como técnica de evaluación de las tecnologías de la información (TIC). Revista de Tecnología de In- formación y Comunicación en Educación, 7, 11-22.

Collin, R., Fredericq, S., Freshwater, D.W., Gilbert, E., Madrid, M., Maslakova, S., Miglietta, M.P., Rocha, R.M., Rodríguez, E. \& Thacker, R.W. (2016). TaxaGloss - A Glossary and Translation Tool for Biodiversity Studies. Biodiversity Data Journal, 4: e10732.

Concepción, M. (2014). Los mapas conceptuales: influencia en el pensamiento creativo del profesor en la asignatura Historia de Cuba. Revista Electrónica EduSol, 14: 1-12.

Crespo, T. (2007). Respuestas a 16 preguntas sobre criterio de Expertos. Editorial San Marcos. Perú, 2007. 117 pp.

DHAMM (Declaración de Helsinki de la AMM). (2013). Principios éticos para las investigaciones médicas en seres humanos. 64a Asamblea General, Fortalez, Brazil, octubre. World Medical Association, Inc. - All Rights reserved. 9 pp.

DRAE (Diccionario general ilustrado de la lengua española). (2012). Barcelona, España. https://www.google. com.cu/?gws_rd=cr\&ei=Pp9OWJGXEY2qjwOs8ovQDQ\#q=Qu\%C3\%A9+es+un+diccionario. [Consultado 12/12-2019].

Ferreira, A. \& González, E. (2000). Reflexiones sobre la enseñanza de la Física Universitaria. Enseñanza de las Ciencias, 18, 189-199.

Fellowes, M. (2020). 30-Second Zoology: The 50 most fundamental categories 
and concepts from the study of animal life. Ivy Press. 160 p.

García, I. \& Fernández, S. (2008). Procedimiento de aplicación del trabajo creativo en grupo de expertos, Energética, 29, 46-50.

Holley, D, 2017. General Zoology: Investigating the Animal World. Dog Ear Publishing. 818 p.

ICZN (International code of zoological nomenclature). (2003). $4^{\text {th }}$ Ed. Published by The International Trust for Zoological Nomenclature. London. $106 \mathrm{pp}$.

ICZN (International code of zoological nomenclature). (2020). Proposed Amendments to the Constitution of the International Commission on Zoological Nomenclature. ZooKeys, 931, 1-9.

Kinchin, I.M. (2010). If concept mapping is so helpful to learning Biology, why aren't we all doing it?. International Journal of Science Education, 23: 1257-1269.

Latin, K., Merdić, E. \& Labak, I. (2016). Concept maps as a tool for better learning Biology in High School. Educatio Biologiae, 2: 1-12.

Mengual, S. (2011). La importancia percibida por el profesorado y el alumnado sobre la inclusión de la competencia digital en educación Superior (Tesis Doctoral). Universidad de Alicante. España, (s/p).

Miller, S. \& Harley, J. (2015). Zoology. Gyldendal, Nordisk Forl. 640 p.
Moulines, C. (2017). Fundamentos de Filosofía de la Ciencia. Alianza Editorial. p. 21.

Salinas, J. (2007). Modelos mixtos de formación universitaria presencial y a distancia: el Campus Extens. Cuadernos de Documentación Multimedia. http://www.ucm.es/info/ multidoc/multidoc/revista/Cuad67/Salinas.html.

Vlachos, E. (2019). Introducing a new tool to navigate, understand and use International Codes of Nomenclature. PeerJ, 7, e8127.

Zayas, P. (2011). El proceso del análisis y la descripción con las especificaciones para confeccionar la matriz de las competencias y construir el perfil del cargo o de ocupación. Ejemplo de dependiente gastronómico en la rama turística. Revista de Investigación y Desarrollo Local, 4(9), [Consultado el 27 de agosto de 2020 de: http://www.eumed.net/rev/turydes/09/pmza.html

Zumbado, F.H. (2004). Modelo didáctico de un Libro de Texto en formato electrónico para la asignatura Análisis Químico de los Alimentos I, en la Carrera de Ciencias Alimentarias. La Habana, Cuba, 217 pp.

Zuev, D.D. (1987). El Libro de texto escolar. Editorial Pueblo y Educación. http://cict.umcc.cu/repositorio/ directorio_eventos/ENFIQUI\%20 2016/res/Rafael\%20Arminana\%20 LIBRO\%20DE\%20TEXTO.pdf. [Consultado el 20 de enero de 2019]. 
